\title{
Traffic differentiation - a basic step towards providing end-to-end QoS on the train-to-wayside wireless communication system
}

\author{
Milos Rovcanin, Dries Naudts, Daan Pareit, Johan Bergs, Erwin Van de Velde, Ingrid Moerman, Chris Blondia
}

\begin{abstract}
We developed a network platform that is responsible for an uninterrupted and seamless connectivity from the train to the wayside through heterogeneous wireless access technologies. However, limiting the offered services to only an onboard Internet is not a feasible business case. A viable one should extend to a broad spectrum of railway communication services like: train control, diagnostics, real time passenger information, entertainment, security CCTV surveillance etc. In a highly volatile environment (from the communication link point of view) such a fast moving train, it is neccesary to introduce prioritization among different traffic classes. This will implicitly determine under what conditions a certain flow should get suspended or dropped in order to preserve the flows of a higher priority as long as possible and to ensure that they meet their QoS demands. The first step towards this goal is data traffic differentiation.
\end{abstract}

Index Terms - Data traffic differentiation, Click Modular Router, IPv6, railway

\section{INTRODUCTION}

A communication system between fast moving trains and the ground involves some significant challenges [1], which are mainly caused by a very dynamic behavior of communication channels due to high speed of a train. Doppler shifts, variation in line-of-sight (LoS) between train and base stations, frequency selective fading, handover etc. are the most notable ones. They cause variations in conditions of communication channels that are both spacial and temporal.

Previous research [2,4] suggested simultaneous use of three different types of wireless communication technologies (satellite links, wireless local area networks, mobile operator networks) in order to provide an uninterrupted and seamless connectivity between train and wayside. A Satellite link could be used as the main communication channel, with a backup in public 2G/3G networks when there is no LoS. WiFi communication would be used when the train is situated in a railway station.

In order to guarantee a user friendly experience, it is inevitable to provide good Quality of Service (QoS) mechanisms in the network architecture. Specific QoS research is performed in IEEE wireless technologies, such as

October, 2011

M. Rovcanin, D. Naudts, D. Pareit and I. Moerman, Department of Information Technology, Internet Based Communication Networks and Services, Ghent University, B-9050 Gent, Belgium. (Phone: +3293314946 ; e-mail: milos.rovcanin@intec.ugent.be, dries.naudts@intec.ugent.be, daan.pareit@intec.ugent.be, ingrid.moerman@intec.ugent.be)

E. Van de Velde, J. Bergs and C. Blondia, PATS Research Group, Dept. Mathematics and Computer Science, University of Antwerp, Middelheimlaan 1, B-2020 Antwerpen, Belgium (Phone: +3232653519; email: erwin.vandevelde@ua.ac.be, johan.bergs@ua.ac.be, chris.blondia@ua.ac.be) 802.11e, 802.16e [9] and network protocols by the IETF, e.g. Diffserv, IntServ, RSVP. In literature, there is already a research covering QoS in wireless heterogeneous networks [3]. However, fast changing wireless characteristics during train mobility and the use of different wireless technologies still poses some huge challenges in this domain. Moreover, the requirements of the on-board applications can rapidly alter due to the fact that the number of users/clients and applications can quickly change. Thus, it is still a big challenge to provide an adequate integrated QoS solution that can deal both with a heterogeneous and dynamic network environment and dynamic application demands.

In Section II we will describe the general architecture of the system we designed with the focus on the main modules used for data traffic differentiation. Other modules are not directly involved in the above mentioned process, therefore they are out of the scope of this paper and will not be described here. Section III will present the experiments we conducted while the results are presented in Section IV. Section V includes future work and a conclusion.

\section{GENERAL ARCHITECTURE}

We designed [6][14] a new and modular architecture for the Train-To-Wayside-Control-System (TWCS). All traffic flows from the Mobile Control Equipment (MCE) - a standard onboard gateway for all the outgoing traffic, to the Wayside Control Equipments (WCE) at the wayside, through the modules of the data plane, which is depicted in Fig. 2. A differentiation is made between connections for reliable transport (straight lines), e.g. TCP (Transmission Control Protocol) connections, and connections for unreliable transport (dotted lines), e.g. UDP (User Datagram Protocol) streams, as they have different requirements. Elements in the control and signaling plane are depicted on Fig. 1. These elements provide configuration information and process control information which is needed during the operation of the data modules.

Within the TWCS communication system we aim at offering an optimized connected experience by prioritizing important traffic flows, enforcing Service Level Agreement (SLA) levels, respecting traffic flow characteristics (e.g. low latency), traffic shaping according to available bandwidth etc. This is jointly referred to as the QoS aspect of the system. The functionality is logically split into the Marker, SLA Enforcer, Shaper and Scheduler elements and they are presented as the part of a data plane. Firstly, the Marker marks packet flows with a service class and priority by using the DiffServ architecture [7], according to different services and their flow characteristics. IETF Differentiated Services (DiffServ) architecture is used for classification within the 


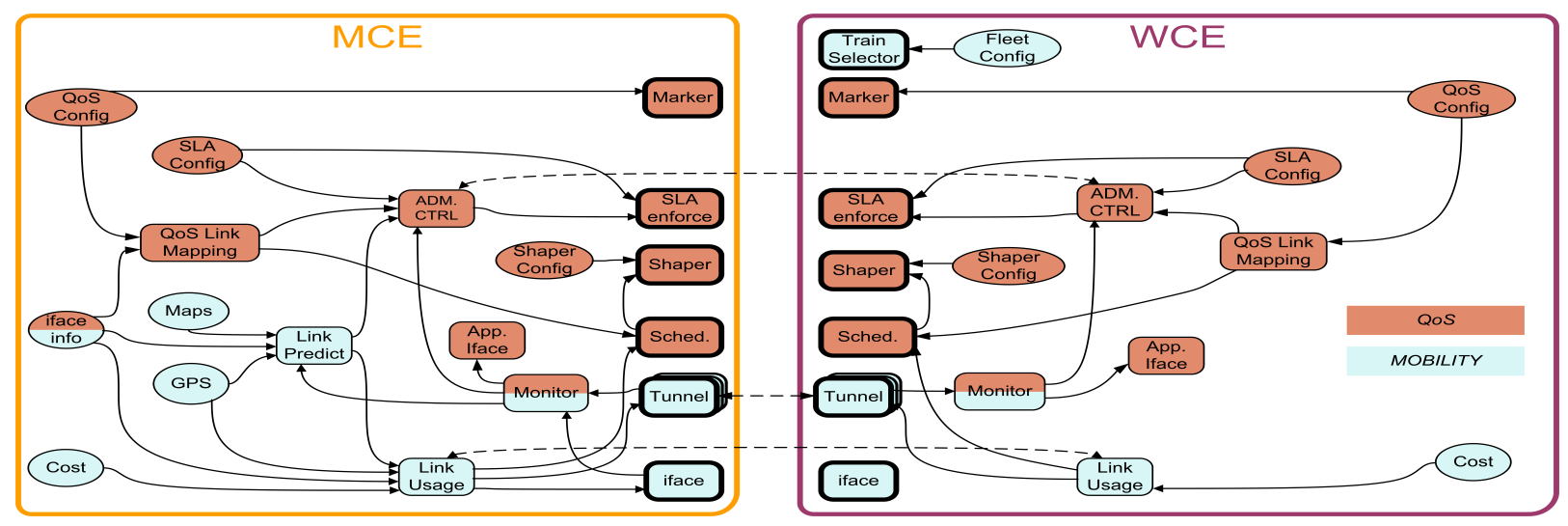

Fig. 1. General architecture - control plane

TWCS. This is a set of enhancements to the Internet protocol to enable QoS between hosts in different networks. Traffic is classified into a limited set of service classes which are

Area Network (VLAN). This way, SLA identification is indicated in the VLAN header.

By adding a unique flow label, VLAN tag and DSCP marker to the IPv6 packet header, the process of data traffic differentiation is completed. A packet will be send towards

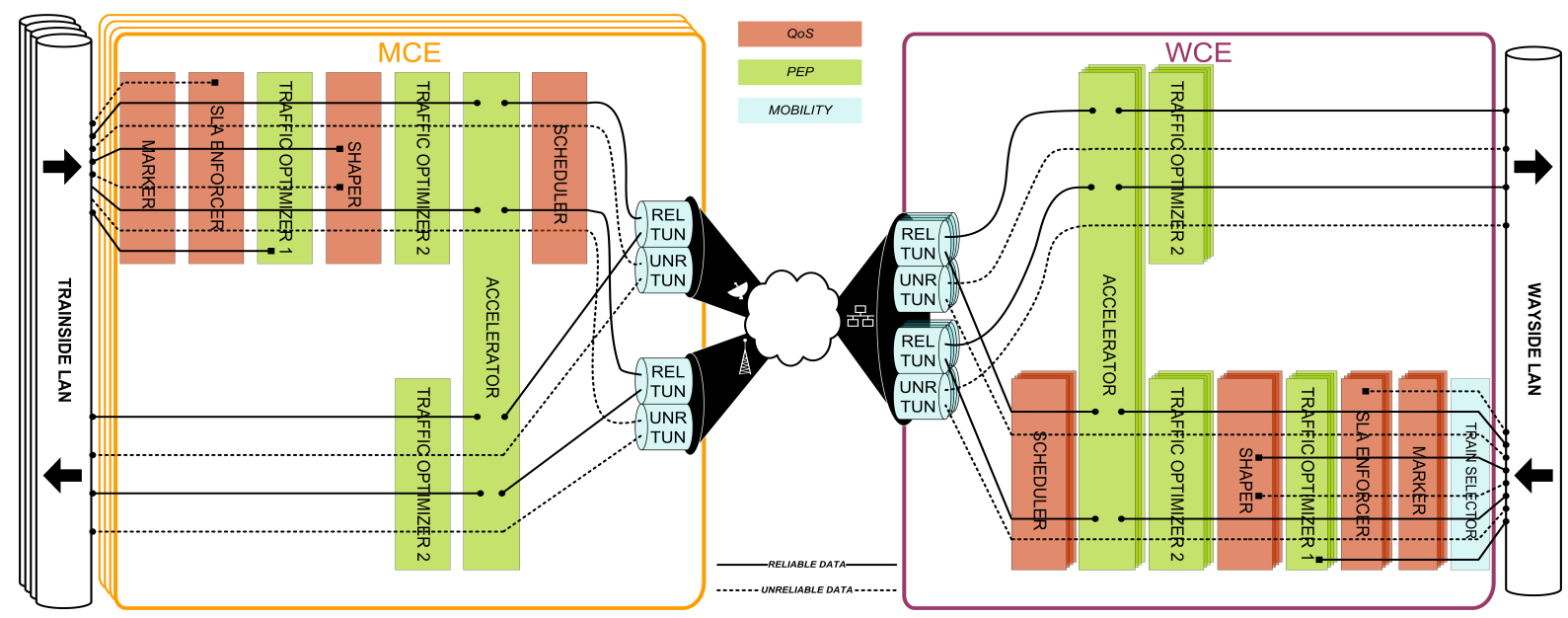

Fig. 2. General architecture - data plane

treated differently. According to characteristics of the trainto- wayside (T2W) services, they are categorized in different 'service classes' (i.e. data traffic that requires specific delay, jitter and loss characteristics from the network), as stated in Table 1 . Additionally, Table 2 presents a relative priority of the T2W services.

Every data flow (a sequence of IPv6 packets from one host to another) is marked with the unique flow label (20 bits) value in a packet header. The packets will also be assigned the Differentiated Service Code Point (DSCP) marker (6 bits) of the IPv6 Traffic Class bits (8 bits) in the IPv6 header [5]. This value indicates into what service class the packets are classified to and what priority they have (Table I). The other two bits of the Traffic Class field are used for Explicit Congestion Notification (ECN). Next, the SLA Enforcer ensures that all flows that belong to the same SLA comply to the SLA stipulations (e.g. maximum data rate, data volume). Every device is subjected to a certain SLA. This agreement can e.g. restrict the type of services that a device is allowed to use. Within this architecture, all devices that are subject to the same SLA are put into a separate Virtual Local the Shaper and Scheduler elements. Then, the Shaper will shape all flows to the available capacity on the wireless trainto-wayside link by dropping packets of flows, with respect to the relative priority of the different flows. Finally, the Scheduler needs to schedule all flows to an appropriate link, considering the service class of each flow (e.g. low latency

TABLE I

DSCP BITS FOR T2W SERVICES

\begin{tabular}{r|cc|c|c} 
Services & Class & Priority & DSCP & Traffic Class \\
\hline Passenger Internet & $\mathrm{A}$ & 1 & 000011 & $00001100(0 \mathrm{x} 0 \mathrm{C})$ \\
\hline Crew Intranet & $\mathrm{A}$ & 2 & 000111 & $00011100(0 \times 1 \mathrm{C})$ \\
\hline Diagnostics & $\mathrm{A}$ & 3 & 001011 & $00101100(0 \times 2 \mathrm{C})$ \\
\hline $\begin{array}{r}\text { Application update } \\
\text { Content update }\end{array}$ & $\mathrm{A}$ & 4 & 001111 & $00111100(0 \mathrm{x} 3 \mathrm{C})$ \\
\hline TCMS event & $\mathrm{B}$ & 4 & 010011 & $01001100(0 \times 4 \mathrm{C})$ \\
\hline CCTV security & $\mathrm{C}$ & 5 & 010111 & $01011100(0 \times 5 \mathrm{C})$ \\
\hline Intercom (VoIP) & $\mathrm{D}$ & 6 & 011011 & $01101100(0 \mathrm{x} 6 \mathrm{C})$ \\
\hline CCTV safety & $\mathrm{E}$ & 6 & 011111 & $01111100(0 \times 7 \mathrm{C})$ \\
\hline TCMS cyclic & $\mathrm{F}$ & 6 & 100011 & $10001100(0 \times 8 \mathrm{C})$ \\
\hline $\begin{array}{r}\text { Public address } \\
\text { PIS control data }\end{array}$ & G & 7 & 100111 & $10011100(0 \mathrm{x} 9 \mathrm{C})$ \\
\hline Configuration traffic & G & 8 & 101011 & $10101100(0 \times \mathrm{xAC})$
\end{tabular}


requirement for VoIP). The flow is finally passed to the Performance Enhance Proxy (PEP) element and sent towards the wayside using the Stream Control Transmission Protocol (SCTP) [15].

Just like the Marker and SLAEnforcer, both Scheduler and Shaper elements belong to the QoS part of the system, but they are out of the scope of this paper since they do not participate in the process of traffic classification. They use that sort of information to shape the traffic and schedule it to an appropriate link.

\section{IMPLEMENTATION}

Complete implementation has been done using the Click Modular Router 1.8.0 [8]. Basic router functions are already implemented inside the Click's kernel. However, most of the specific functionalities of our configuration are incorporated inside the elements we developed.

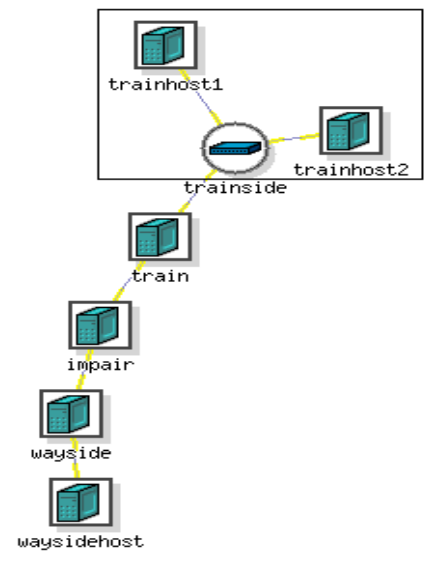

Fig.3. Experimental network topology

The IBBT iLAB Virtual Wall testbed $[10,11]$ was used for the purpose of testing and evaluation. Six PCs, each with 3 or 4 gigabit ethernet interfaces, were organized into a small network as depicted in Fig. 3. "Trainhost1" and "Trainhost2" simulate onboard hosts. "Train" is an onboard router, while "Wayside" is its counterpart on the ground. "Waysidehost". The "Impair" node is used to run the scripts that should change the properties of available communication links, simulating fast movement of a train and changes links suffer according to it.

Linux Kernel 2.6.39 with latest SCTP developments was mounted on each of the PCs. For the purpose of generating data flows, we used Jperf 2.0.2 [12] and a VLC streamer [13].

Details about the implementaion were previously presented [6]. The most notable adjustments we made are:

- Instead of using the IEEE 802.1q protocol, better known as VLAN tagging, we developed an element that sets up VLAN designators inside a Flow Label field of an Ipv6 header. VLAN tags are added according to the source address of packet and a VLAN to SLA mapping is predefined and statical.
- The Marker element decides upon a flow label by inspecting n-tuple of parameters in the packet header, typically including IP source address, IP destination address, source port number, destination port number and protocol identification. Every new flow label is set by means of a pseudo-random generator.

- The DSCP designator for each packet is usually determined on the application level. In the absence of it, we agreed to use a destination port of a flow to decide what traffic class it belongs to (see Table II).

TABLE II

DESTINATION PORT TO TRAFFIC CLASS MAPPING

\begin{tabular}{|l|l|l|l|l|}
\hline Port range* $^{*}$ & DSCP** & Traffic Class IP6 field** & Traffic Class & Priority \\
\hline $0-1100$ & 3 & $00001100(0 \times 0 C)$ & A & 1 (lowest) \\
\hline $1101-1200$ & 7 & $00011100(0 \times 1 C)$ & A & 2 \\
\hline $1201-1500$ & 11 & $00101100(0 \times 2 C)$ & A & 3 \\
\hline $1501-2000$ & 15 & $00111100(0 \times 3 C)$ & A & 4 \\
\hline $2001-3000$ & 19 & $01001100(0 \times 4 C)$ & B & 4 \\
\hline $3001-3500$ & 23 & $01011100(0 \times 5 C)$ & C & 5 \\
\hline $3501-4000$ & 27 & $01101100(0 \times 6 C)$ & D & 6 \\
\hline $4001-4200$ & 31 & $01111100(0 \times 7 C)$ & E & 6 \\
\hline $4201-4500$ & 35 & $10001100(0 \times 8 C)$ & F & 6 \\
\hline $4501-5000$ & 39 & $10011100(0 \times 9 C)$ & G & 7 \\
\hline $5001-99999$ & 43 & $10101100(0 \times A C)$ & G & 8 (highest) \\
\hline
\end{tabular}

\section{RESULTS}

\section{A. Experiment 1 - introducing traffic classes}

The purpose of this experiment was to demonstrate the

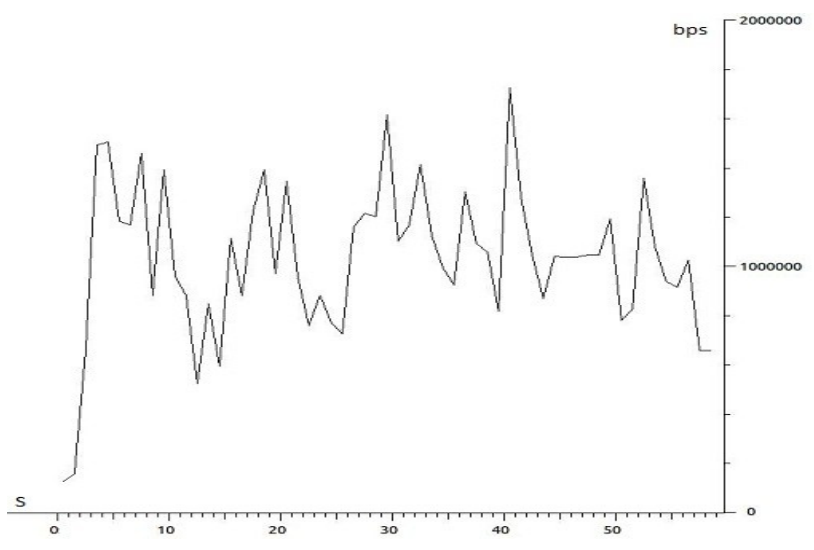

Fig.5. VLC flow trace graph - no link emulation and background flow

impact of data traffic differentiation as opposed to a case when no differentiation is applied.

Click scripts (router configurations) were run on both Train-side and Wayside nodes (see Fig.3). The Impairment node was used to emulate the link between a train and a wayside. During this experiment, only one communication link was active with the dedicated bandwidth of $1.7 \mathrm{Mbps}$, delay $200 \mathrm{~ms}$ and a $0.05 \%$ packet loss. A background UDP 
traffic of $1 \mathrm{Mbps}$ was used. It's direction was from the Trainhost1 towards the Waysidehost. The second flow was a VLC video stream sent from the Waysidehost towards the Trainhost2 (Fig.5). The recorded average rate of the flow was 1.045 Mbps.

\section{A. 1 No traffic prioritization}

In the first case, both flows belonged to the same traffic class. This way, Shaper and Scheduler treated them the same way - neither of the two had the priority over the other one. The experiment was 40 seconds long. A trace of the VLC video stream was made on the network interface of the Trainhost2. The results are depicted on Fig.6.

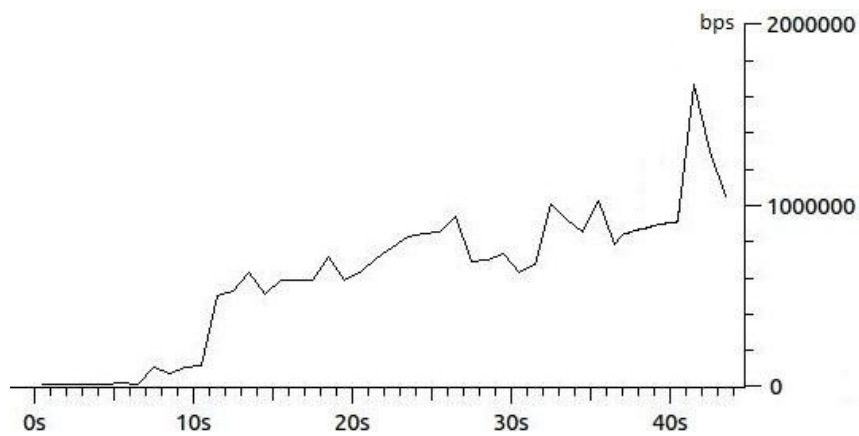

Fig.6 VLC flow trace graph - the same traffic class for both flows

As seen from the graph, the average rate of the VLC stream was significantly bellow $1 \mathrm{Mbps}$. Transmission trace file showed an avrage of $680 \mathrm{Kbps}$ and a $26.32 \%$ packet loss. Jperf statistics of the background flow that the average rate of the background flow was around $780 \mathrm{Kbps}$ with $11 \%$ packet loss. As expected, both flows got as much bandwidth as possible. The slow increase of a flow's rate, that can be observed at the beginning of almost every experiment, was caused by the queuing effects inside Click and a slow start windowing mechanism, introduced by the SCTP tunnels.

A. 2 With traffic class prioritization

In this case, the VLC flow was assigned a higher

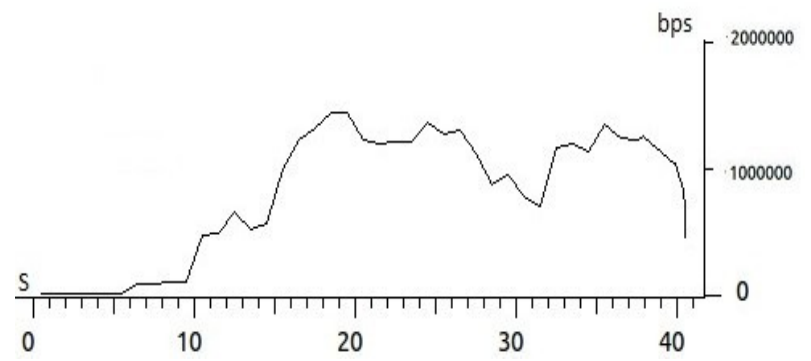

Fig.7. VLC flow trace graph - different traffic classes

priority by sending it to a higher destination port (3600 Intercom, see Table II). The Random Early Detection (RED) elements inside the Shaper started shaping the flows differently, depending on the state of the incoming queue and settings assigned to a dedicated RED [7]. The results are depicted on Fig.7. It can be observed from a graph that the average rate of the VLC flow was significantly increased, to
944 Kbps with 2.35\% packet loss. Jperf statistics displayed a significant decrease of a background flow rate - $584 \mathrm{Kbps}$ with $36 \%$ packet loss.

\section{B. Experiment 2 - changing link conditions}

The setup used for this link was the same as in the Section A2, but this time the bandwidth and delay of a communication link were changed on the fly. This way we tested how the system responses to a sudden change of the bandwidth of a communication link.

\section{B. 1 High bandwidth oscilations}

In the first case a bandwidth of a link was changed from 1Mbps to 1.7 Mbps and finally 2.5 Mbps on the 20s intervals. A trace of the VLC video stream is shown on Fig.8.

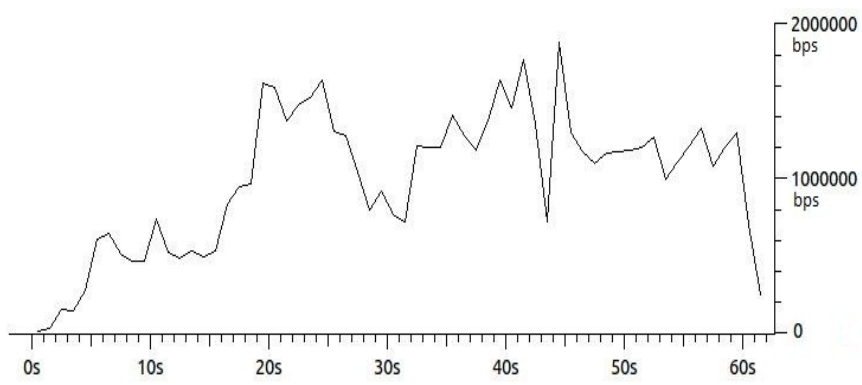

Fig.8. VLC trace graph - less frequent, high link bandwidth oscillations

It can be observed that, after approximately 20 seconds, a flow rate will increase to a value it reached in the second case of the Experiment 1, which was expected since the parameters of the Shaper and the communication link were the same. The next increase, at 40th second, is not as noticeable since the VLC flow rate does not surpass 1.4 Mbps at any moment after the 40th second (see Fig.5).

\section{B. 2 Much lower bandwidth oscilations}

In this, more frequent bandwidth changes were introduced. However, they were not as high as in the first case. These oscillations were introduced in a fashion presented in Table III. The results of the experiment are depicted on Fig.9.

Table III

Low and frequent bandwidth oscillations

\begin{tabular}{|l|c|c|c|c|c|c|c|c|}
\hline Time[s] & $0-6$ & $6-13$ & $13-22$ & $22-28$ & $28-35$ & $35-40$ & $40-45$ & $45-60$ \\
\hline Bandwidth & 1000 & 1400 & 1200 & 900 & 800 & 500 & 400 & 2000 \\
\hline
\end{tabular}

As the graph shows, it is hard to notice small changes in a bandwidth. For example, at 6th, 13th and 40th second no significant difference in the flow rate can be seen, since the oscilations in the available link bandwidth are small. For an ilustration: at the 13th second, the bandwidth changed from 1400 down to $1200 \mathrm{Mbps}$ and the change in average flow rate was $810.26 \mathrm{Kbps}$ to $658.47 \mathrm{Kbps}$ on average flow rate, respectivelly. however, at 22nd, 35th and 45th second, these jumps are much easier to notice. 


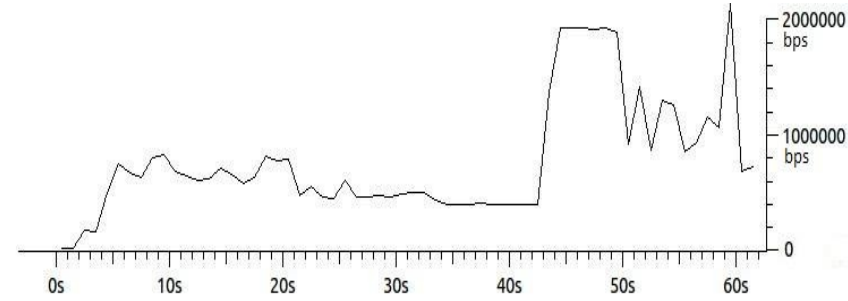

Fig.9. VLC stream graph - More frequent, low link bandwidth oscillation

The most interesting section of the graph is the interval between 45th and 50th second. Since the bandwidth was too low for the flow to be transmitted at at its rate, the incoming queue at the Shaper was filled up to the top. After the bandwidth had suddenly jumped up to 2 Mbps, the queue immediately started to empty itself at almost constant rate (45-49 sec).

\section{B. 3 Link delay oscilations}

In the third case a delay of the link was being changed. We switched between $200 \mathrm{~ms}, 10 \mathrm{~ms}$ and $500 \mathrm{~ms}$, respectively, on a 20 seconds basis. The result is presented on Fig.10. Though it is not noticeable on the graph, the changes of the link delay can be seen on the video itself in a form of glitches and a block effect. These effects last significantly longer when switching from $10 \mathrm{~ms}$ to $500 \mathrm{~ms}$ than during the first transition, $200 \mathrm{~ms}$ to $10 \mathrm{~ms}$.

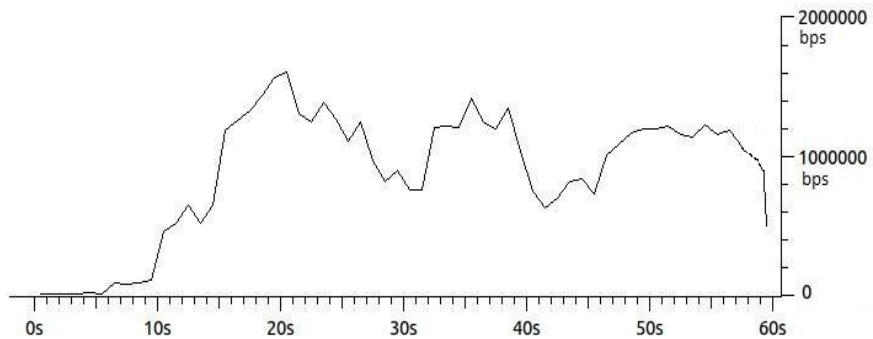

Fig.10. VLC stream graph - link delay variations

\section{Experiment 3 - system response time}

The final experiment was performed in order to show how fast our system can respond to a link bandwidth change.

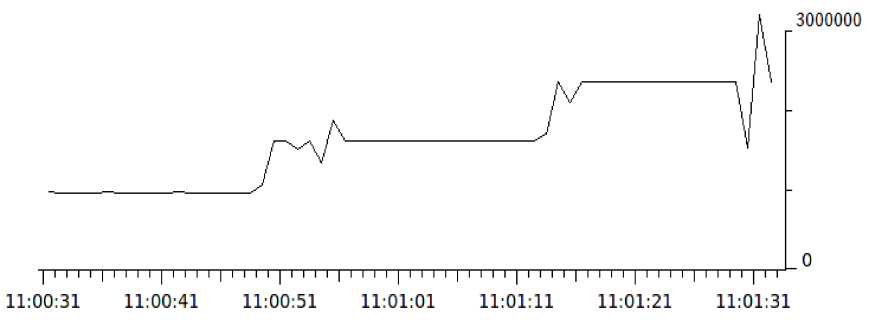

Fig.11. System response time

To make the results as clear as possible we used one UDP flow of a 5Mbps rate and emulated a communication link by changing its bandwidth from $1 \mathrm{Mbps}$ to $1.7 \mathrm{Mbps}$ and finally to 2.5 Mbps. Perl script, used to set up a parameters of the link, also set up time stamps to note down the exact moment when the bandwidth switched from one value to another. The results are depicted in Fig.11. Comparing the values shown on the graph and time-stamp values generated by a Perl script we can conclude the following:

Start-up time stamp - T11:00:30 matches the starting time of the experiment. According to following time-stamps, link bandwidth switches occurred at T11:00:50 (1 Mbps to 1.7 Mbps) and at T11:01:14 (1.7 Mbps to 2.5 Mbps). Compared to the values marked on Fig.11, we can see that the system response time is within one second.

\section{Conclusion}

The basic step, in order to ensure sufficient QoS in the system we proposed, is a proper traffic class differentiation. This concept will ensure, for example, low latency to critical network traffic such as voice or streaming media, while providing simple best-effort service to non-critical services such as web traffic or file transfers.

The next step will be to design a mechanism that will dynamically change the shaping policy according to a current state of the incoming queue, status of a communication link, number of flows and their priorities. These two steps will eventually lead to the main goal and that is to ensure sufficient end-to-end QoS in a volatile environment such as the fast moving train.

\section{Acknowledgement}

This research was carried out as part of the IBBT TRACK ICON project

\section{REFERENCES}

[1] W. van Brussel, "Bringing ICT services to trains: technical and economical challenges" 9th Conference on Telecommunications Internet and Media Techno Economics (CTTE), 2010, 1 -7

[2] B. Lannoo, J. Van Ooteghem, D. Pareit, T. Van Leeuwen, D. Colle, I. Moerman and P. Demeester, "Business model for broadband internet on the train", The Journal of The Institute of Telecommunications Professionals, 2007, 1, 19-27

[3] D.T. Fokum, V.S. Frost, "A Survey on Methods for Broadband Internet Access on Trains," Communications Surveys \& Tutorials, IEEE , vol.12, no.2, pp.171-185, Second Quarter 2010.

[4] L. Verstrepen, W. Joseph, E. Tanghe, J. Van Ooteghem, B. Lannoo, M. Pickavet, L. Martens and P. Demeester, "Making a well-founded choice of the wireless technology for train-to-wayside data services," 9th Conference on Telecommunications Internet and Media Techno Economics (CTTE), 2010, 1-7

[5] S. Deering, R. Hinden. Internet Protocol, Version 6 (IPv6) Specification (1998). URL http://tools.ietf.org/html/rfc2460

[6] M. Rovcanin, D. Pareit, D. Naudts, J. Bergs, E. Van de Velde, I. Moerman, C. Blondia, "Data traffic differentiation and QOS on the train, in fast parameter varying, heterogenous wireless networks", IEEESCVT 2011, Gent, Belgium

[7] J. Babiarz, K. Chan, F. Baker. Configuration Guidelines for DiffServ Service Classes (2006). URL http://tools.ietf.org/html/rfc4594

[8] E. Kohler, The Click Modular Router. Ph. D. thesis, Massachusetts Institute of Technology (2001).

URL http://pdos.csail.mit.edu/papers/click:kohler-phd/thesis.pdf

[9] Lim, W., Kim, D., Suh, Y., and Won, J. 2009. Implementation and performance study of IEEE 802.21 in integrated IEEE 802.11/802.16e networks. Comput. Commun. 32, 1 (Jan. 2009), 134-143.

[10] URL www.ibbt.be/en/develop-test/ilab-t/virtual-wall

[11] URL http://www.emulab.net

[12] URL www.rarts.net/software/jperf

[13] URL www.videolan.org/doc/streaming-howto/en

[14] Pareit, D. et al; A novel network architecture for train-to-wayside communication with Quality of Service over heterogeneous wireless networks; in press for EURASIP Journal on Wireless Communications and Networking, 2012.

[15] http://tools.ietf.org/html/rfc4960 
